\title{
Experimental and modelling buckling of wood-based columns under repeated loading
}

\author{
F. Bouras ${ }^{1}$, M. Chaplain ${ }^{2}$ and Z. Nafa ${ }^{1, \mathrm{a}}$ \\ ${ }^{1}$ LGCH, Université de Guelma, PB401- 24000 Guelma, Algérie \\ ${ }^{2}$ US2B, Université Bordeaux 1, 351 cours de la libération- 33405 Talence cedex, France
}

\begin{abstract}
Collapse of timber constructions can appear under the effect of load that exceeds the resistance of a carrying element or under the effect of a geometrical instability like buckling. In addition, loading can be constant or varying for example loads due to wind or earthquakes. The aim of this paper is to study the behaviour and the lifetime of columns in wood or based-wood material such as glulam (GL) or laminated veneer lumber (LVL) under repeated loading leading to buckling.
\end{abstract}

\section{Introduction}

Despite the large use of wood in element of construction subject to buckling as columns, studies on this behaviour are very limited. In static behaviour, we can cite the work of Fairker [1], wood columns of various lengths in two species were evaluated for strength and the results analyzed by several methods. The data thus obtained were used to predict buckling loads given by the tangent modulus theory. In one species, the average experimental results for short and intermediate columns of the grade used agreed very closely with the tangent modulus predictions. In the other species, average experimental results went above tangent modulus predictions for the short columns and below for the intermediate columns. The approximate theoretical tangent modulus buckling loads of the columns with L/d equal to 15 and 20 were determined by Southwell's method. The results that were obtained by Southwell's method agreed very closely with theory for intermediate columns of one species, but they were too high for intermediate columns of the other species. Agrawal [2] carried out an experimental work on solid timber columns of small cross-sections. The variables investigated include breadth to depth ratio, and wide range of slenderness ratio, one each, of conifers and hardwoods. The study indicates that imperfections in material, initial curvature of strut and eccentricity of loading can be considered by using a proper value of $\eta$ in Perry's formula. Reduction factors for evaluating the strength of such struts for Indian timbers have been reported.

Studies on cyclic buckling behaviour of wood or wood-based columns are no available. The found papers deal with other materials as concrete, steel or composites. In order to fill this void, we initiate this work. The aim of this paper is to study experimentally the behaviour of columns in wood or based-wood material such as glulam (GL) or laminated veneer lumber (LVL) under repeated loading leading to buckling.

\footnotetext{
a e-mail : naf_zah@yahoo.fr
}

This is an Open Access article distributed under the terms of the Creative Commons Attribution-Noncommercial License 3.0, which permits unrestricted use, distribution, and reproduction in any noncommercial medium, provided the original work is properly cited. 


\section{Experimental procedures}

Specimens studied are realised in solid wood (spruce), glulam (GL28), laminated wood or LVL kerto-S, (Laminated Veneer Lumber). . These specimens are a homothetic to columns which can be found in timber structures. Two sets of 18 pieces of LVL a set of 17 specimens of glulam (GL28) and a set of 36 specimens of solid wood (spruce). The average characteristics, size, density and moisture content are listed in Table 1.

Table 1. Specimens' characteristics (sd : standard deviation).

\begin{tabular}{|l|c|c|c|c|c|c|c|c|c|c|c|}
\hline & \multirow{2}{*}{$\begin{array}{c}\text { nb of } \\
\text { specimens }\end{array}$} & \multicolumn{2}{|c|}{ Width (mm) } & \multicolumn{2}{c|}{ Thickness (mm) } & \multicolumn{2}{c|}{ Length (mm) } & \multicolumn{2}{c|}{ Specific gravity } & \multicolumn{2}{c|}{ Moisture content } \\
\cline { 3 - 14 } & average & sd & average & sd & average & sd & average & sd & average & sd \\
\hline Solide Wood & 36 & 98,9 & 3,2 & 17,4 & 0,1 & 799,8 & 1,2 & 0,440 & 0,032 & $12,3 \%$ & $1,4 \%$ \\
\hline Glulam (GL28) & 17 & 98,0 & 0,7 & 17,5 & 0,2 & 801,5 & 1,3 & 0,481 & 0,018 & $12,1 \%$ & $0,4 \%$ \\
\hline LVL(Kerto-S) & 36 & 99,9 & 0,7 & 17,4 & 0,3 & 798,3 & 3,8 & 0,542 & 0,023 & $9,5 \%$ & $1,7 \%$ \\
\hline
\end{tabular}

The joints of the mechanism of buckling are composed of two half-cylinder shaped to receive our pieces and inserted in leg angles (Figure 1). This angle is fixed by welding, on a stand which takes place on the testing machine. A Teflon sheet wedged between these two elements allows us to obtain very low friction, thus forming an almost perfect articulation. The buckling length is thus equal to the length of the specimen.
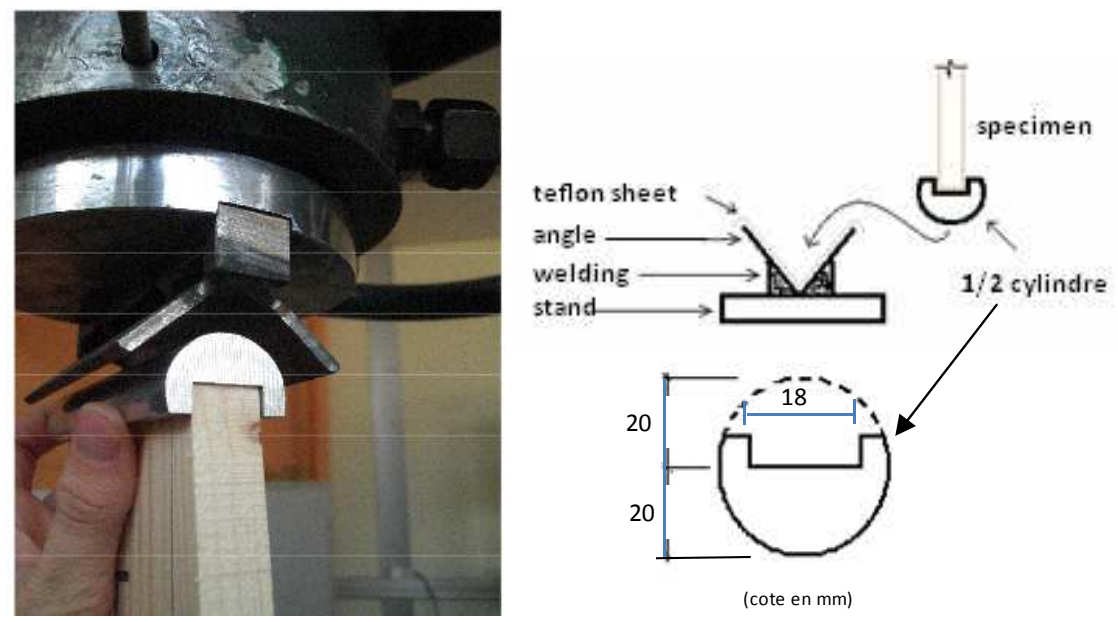

Fig. 1. Buckling device.

During testing, the time, the displacement of the crosshead of the machine, the compressive force applied and the eccentricity of the beam in the middle are measured. The record of the eccentricity was performed using a displacement sensor (LVDT - stroke $65 \mathrm{~mm}$ ) and a $20 \mathrm{~m}$ course laser sensor which took over the position where the LVDT was out or race (figure 2). The measurement of this eccentricity will allow us to calculate the maximum moment (at the middle of the beam) as a result of buckling and thus can deduce the stress state. 


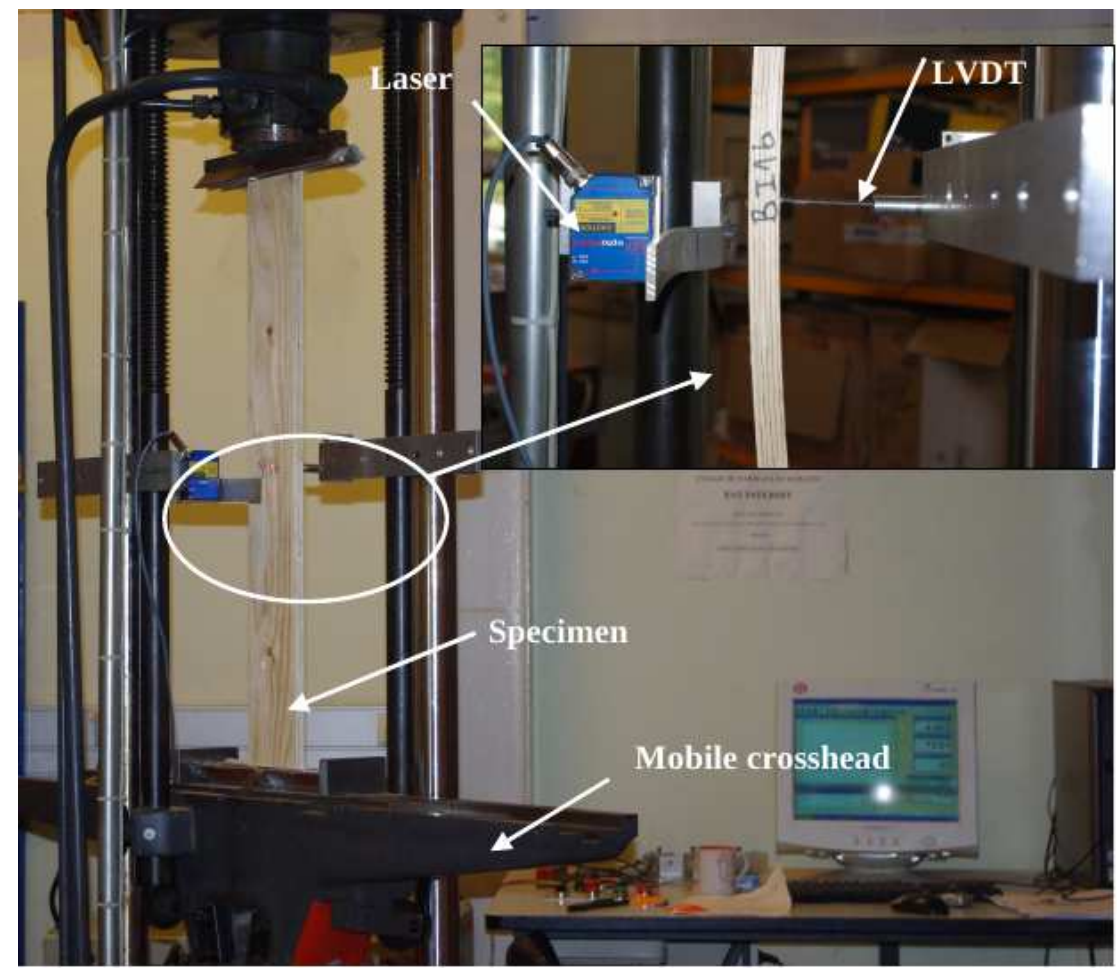

Fig. 2. The 20T hydraulic test machine and measuring device.

\section{Experiments}

\subsection{Monotonous tests}

Before cyclic tests, monotonous -static - tests are conducted to determine the crosshead displacement corresponding to the beginning of instability. This displacement is noted $\delta \mathrm{e}$ and will serve as a reference during cyclic loading led in displacement. The experiments are carried at $1 \mathrm{~mm} / \mathrm{min}$ speed. Figure 3 shows an example of curves load-crosshead displacement. We can clearly distinguish the instability leading to a decrease of the load. Figure 3 also emphasize that the 3 species (wood, GL, LVL) have nearly the same behaviour until the instability. We saw during these trials that there was no significant difference in behaviour between the solid wood, glulam and LVL. 


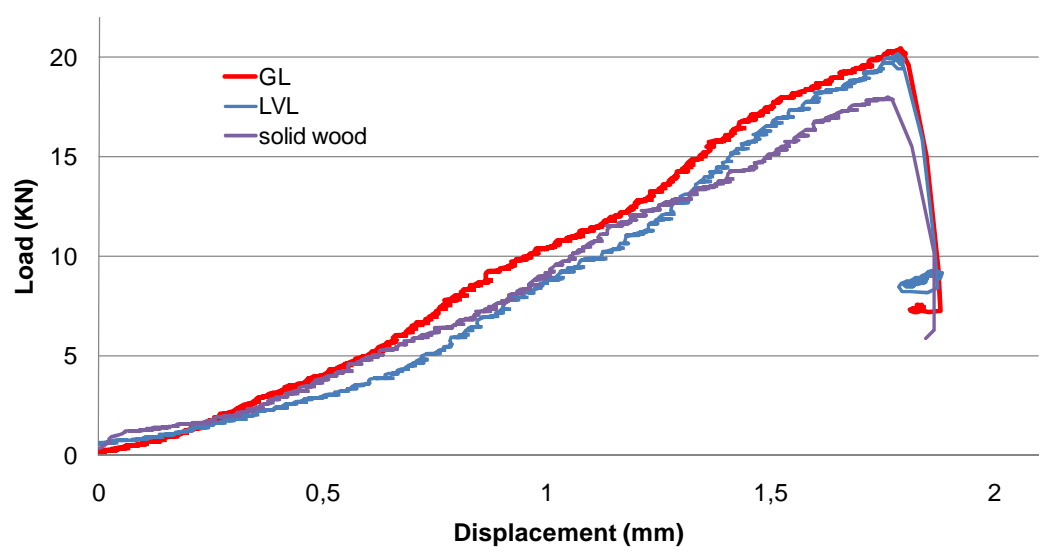

Fig. 3. Force-displacement curves obtained during monotonous buckling tests - Comparison between solid wood, LVL and GL responses.

\subsection{Cyclic tests}

The testing machine is controlled in displacement. The protocol is inspired by those used to simulate seismic actions and in particular those proposed by Ceccotti [3]. The protocol proposed has been amended to facilitate the processing of results and it is a mix between a rapidly growing cycle test and a fatigue test. This protocol has been heavily imposed by the hydraulic testing machine used that does not allow us to make very quick and / or prolonged cycles.

Analysis of monotonous buckling curves allows to the load cycle shape: after a pre-load at a speed of $50 \mathrm{~mm} / \mathrm{min}$ until $\delta \mathrm{e}$ (displacement in the beginning of the instability under static loading), the specimens are submitted to periodic triangular loading with a constant amplitude equal to $0,5 \delta$ e and at a speed of $290 \mathrm{~mm} / \mathrm{min}$ (Figure 4). The cyclic loading is done by "packages" of 50 successive cycles with a gradual increase of $0,5 \delta$ e between packages. In the first package, the maximum

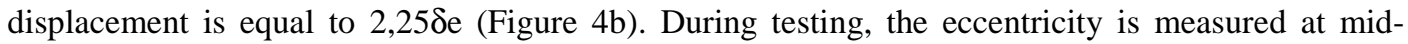
height of the beam. The obtained average value of $\delta \mathrm{e}$ is around $2 \mathrm{~mm}$ for the three species.

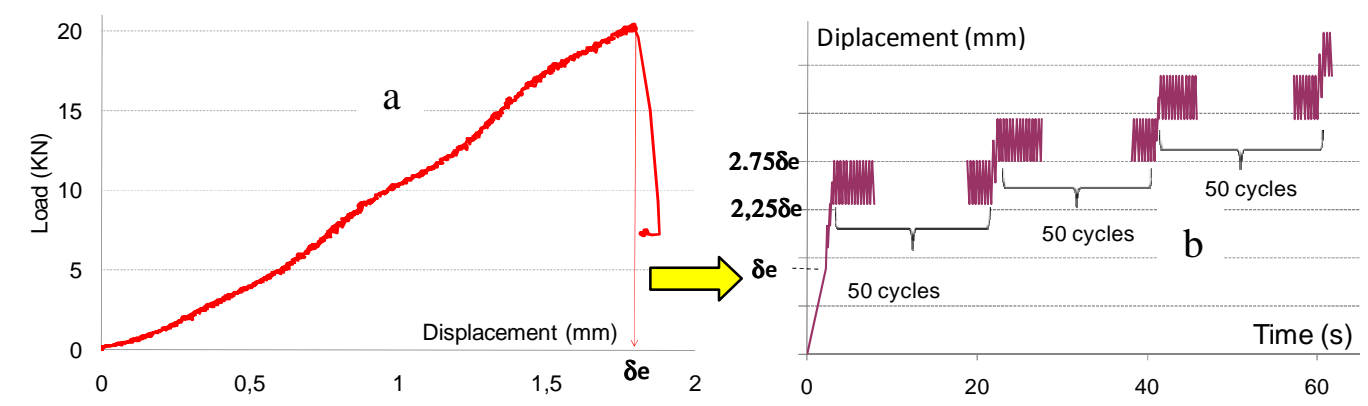

Fig. 4. a) Force-displacement curve obtained through monotonous buckling tests - b) protocol of cyclic buckling tests.

Due to the large inertia of the crosshead, the cyclic order is not respected and the actual displacement is more important: the amplitude of each cycle is not equal to the theoretical value $0,5 \delta_{\mathrm{e}}$ but it nearly equal to $0,9 \delta \mathrm{e}$. Also, the real cyclic period is nearly $50 \%$ greater than the theoretical value. 


\section{Cyclic Results}

During cyclic tests, different failure modes have been found for each species specimens (figure 5):

- Solid wood is first damage in compression usually followed by a prompt failure by tension.

Generally, fracture occurs not in the middle of the specimen but near knots.

- Glulam breaking is similar to solid wood. Generally, one lamella breaks in tension and this induced the failure of the other lamellas.

- LVL is also damaged in compression and the rupture is due to detachment of layers veneers in the tension zone. This failure is mainly localized at middle-height of the beam, the most stressed zone, except when lengthening joints are located near the extreme tension area. In this case, the rupture occurs in this joint.
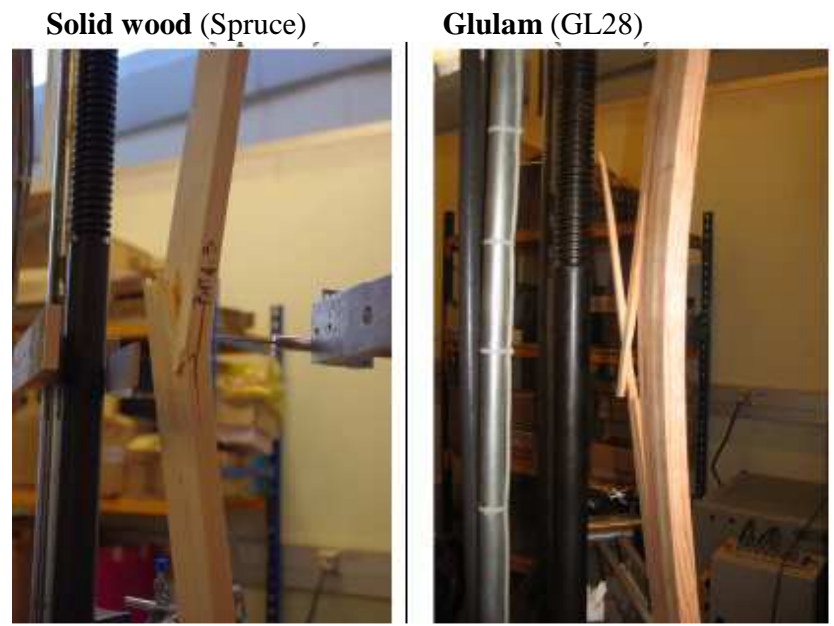

LVL - (kerto-S)

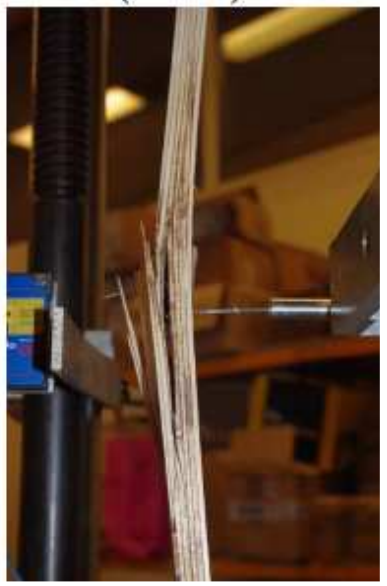

Fig. 5. Failure modes of different specimens' species.

Figure 6a shows an example of load-displacement curves obtained during testing of laminated wood. The curves for other species of material are similar. After instability, when the displacement exceeds the $\delta$ e value, the strength usually half decreases. Then, force continues to decline, by cons, part of the increase in eccentricity, the stresses in the specimen increases until it reaches the limit of the material (Figure $6 \mathrm{~b}$ ). Figure $6 \mathrm{~b}$ shows the evolution of maximum stress in the central part of the beam. We can observe the increase of stress before the brittle fracture by tension.
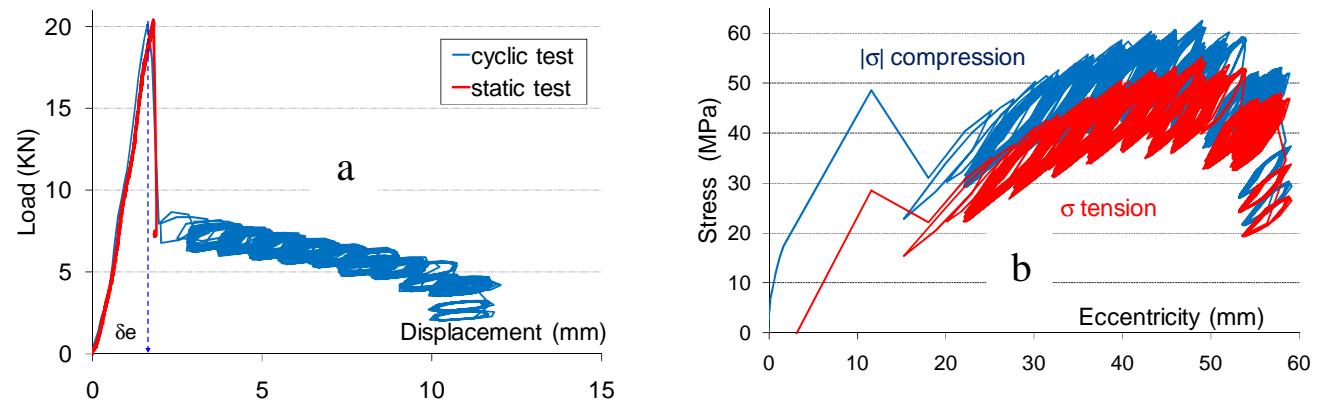

Fig. 6. Example of curve in force vs. crosshead displacement and stress at mid height of the GL beam. 
Table 2 summarised the average maximum compression stress obtained and also the values of the crosshead displacement and of the eccentricity corresponding to this maximum stress. We can note that the eccentricity corresponding to the beginning of the decreasing of the stress is quite the same for the three kinds of specimens. The maximum stress is different because the testing "woods" have different bending strengths. The characteristic strength in bending according to the Eurocode 5 is $28 \mathrm{MPa}$ for the GL28, $48 \mathrm{MPa}$ for the LVL Kerto-S. The using wood has a lot of knots, may be classed as a $\mathrm{C} 18$ (characteristic bending strength $=18 \mathrm{MPa}$ ) or as a C22.

Table 2. Average and standard deviation (sd) of the maximum compression stress and the corresponding displacement crosshead and eccentricity.

\begin{tabular}{|l|c|c|c|c|c|c|}
\cline { 2 - 7 } & \multicolumn{2}{c|}{ Max. Compresssion stress (Mpa) } & at the displacement $(\mathrm{mm})$ & \multicolumn{2}{c|}{ at the eccenticity $(\mathrm{mm})$} \\
\cline { 2 - 7 } & average & $\mathrm{sd}$ & average & $\mathrm{sd}$ & average & $\mathrm{sd}$ \\
\hline Solid Wood & 48,9 & 8,5 & 7,1 & 2,0 & 45,1 & 7,8 \\
\hline Glulam (GL28) & 56,3 & 12,1 & 8,1 & 2,5 & 45,3 & 8,3 \\
\hline LVL (KERTO-S) & 65,0 & 6,7 & 7,5 & 2,0 & 45,8 & 6,4 \\
\hline
\end{tabular}

For solid wood, the number of cycles to failure can be equal to 10 or more than a hundred (up to 300 cycles) depending on the presence or not of knots. For GL, this number of cycles also dependents on the existence of lengthening joints or knots and it can vary between 300 and 500. For LVL, this number can also have a great variation (10 to 500 cycles) if LVL has lengthening joints near the middle of the specimens or not.

\section{Modelling}

The prediction of number of cycles to failure is studied using a damage theory. The damage is characterized by a parameter $\mathrm{D}$ which varies between 0 when the material is not damaged and $\mathrm{D}_{\mathrm{c}}$ when failure appears. We take $D_{c}=1$.

Only non-linear damage evolution and non-linear cumulative damage have been held. The usual damage models do not take into account the influence of frequency, i.e. they do not integrate the viscoelastic behaviour of the material. To bridge this gap, Chaplain [4] has proposed to couple a rheological model with the damage model of Barrett and Foschi [5].

The expression of the damage model is as follows (Eq. 1):

$$
\begin{cases}\frac{d D}{d t}=a \cdot\left(\frac{\left|\sigma_{D}(t)\right|-\sigma_{o}}{\sigma_{s}}\right)^{b}+\lambda D(t) & \text { if } \quad\left|\sigma_{D}(t)\right|>\sigma_{o} \\ \frac{d D}{d t}=0 & \text { if }\left|\sigma_{D}(t)\right|<\sigma_{o}\end{cases}
$$

where $\sigma_{D}(t)$ is the part of the compression stress applied to the Kevin Voigt spring, $\sigma_{s}$ the static compression strength and $\sigma_{o}$ the threshold stress. $a, b$ and $\lambda$ are parameters. |.| means absolute value. As the applied stress is negative, the expression of the stress must be express in absolute value. 
The determination of the damage evolution is obtained considered the change of the slope of compression stress versus the eccentricity curves (fig. 7a). First, the lost of rigidity is formulated as follow:

$$
\mathrm{D}^{\prime}=1-\mathrm{K} / \mathrm{Ko}
$$

Where $\mathrm{K}$ is the slope of the compression stress-eccentricity curve, Ko is the initial slope.

The obtained parameter D' is normalized to 1 to obtain the damage parameter noted D (Fig. 7b).
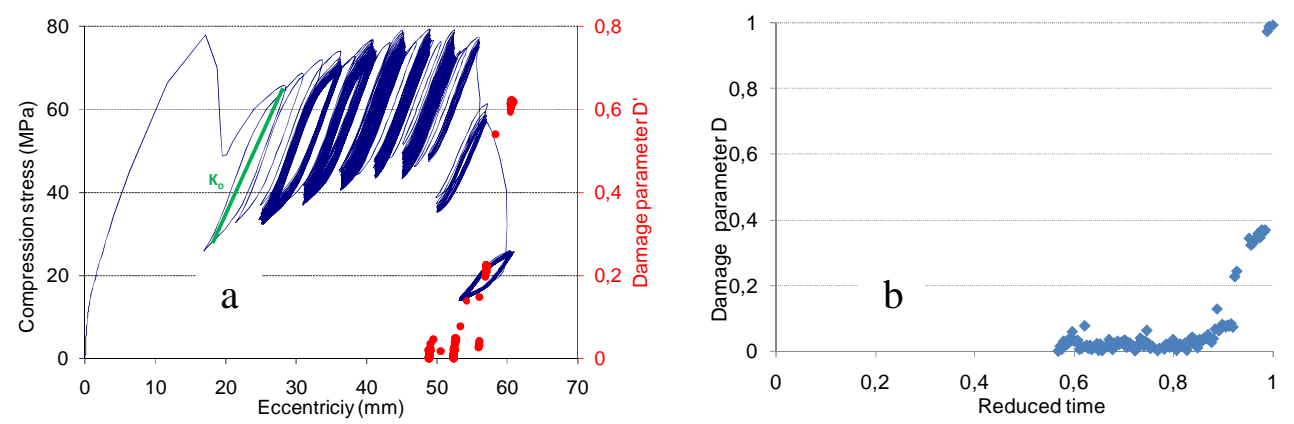

Fig. 7. Determination of the damage parameter D: (a) stress - eccentricity curves and un-normalized damage D'- (b) Damage evolution.

Another way to obtain a damage parameter is to study the evolution of the hysteresis curves. We can observe on fig $7 \mathrm{a}$ that the energy dissipated during a cycle, decreased when the material is damaged.

The tests are not complete yet, we have to carry monotonous tests until failure to estimate the "static" strength $\sigma_{\mathrm{s}}$ of the specimens. Without this static stress, we cannot apply the damage model or performed it.

\section{Conclusion}

In order to analyse the behaviour of wood and wood-based columns under cyclic loading leading to buckling, a set of static and cyclic buckling tests led in displacement was performed on solid wood, glulam and LVL specimens. Behaviour in monotonic test until the instability has appeared quite independent of the specimen specie. The cyclic tests show brittle fracture by traction for solid wood and GL and detachment of layers veneer for LVL. The prediction of number of cycles to failure is studied using a damage theory. A damaged parameter is determinate through the slope change of the compression stress versus eccentricity curves. In order to apply the damage model, further experimental testing will be carrying out.

\section{References}

1. J. E. Fairker, EPL-RN-28, (1964)

2. S. K. Agarwal, S. K. Kaushik, Journal of the Institution of Engineers. India, 70 (1989)

3. A. Ceccotti, Les assemblages bois sous l'action sismique, Structures en bois aux états limites chapitre VIII-2, sujet STEP C17, VIII-2-1 -VIII-2-11, (1997)

4. M. Chaplain, Z. Nafa, M. Guenfoud, Damage and Fracture Mechanics, (Springer, 2009)

5. J.D. Barrett, R.O. Foschi, Canadian Journal of Civil Engineering, 5(1978) 